\title{
A Cost Model For Asynchronous and Structured Message Passing ${ }^{\star}$
}

\author{
Emmanuel Melin, Bruno Raffin, Xavier Rebeuf, and Bernard Virot \\ LIFO - Université d'Orléans, F-45067 Orléans, Cedex 02 - FRANCE \\ Phone: (+33) 238417318 Fax: (+33) 238417137 \\ virot@lifo.univ-orleans.fr
}

\begin{abstract}
We present a cost model that relies on an asynchronous and structured parallel execution model for message passing. We show that it is possible to define a complexity function for programs that yields a symbolic date for each communication event. By ordering these dates, an upper bound for the network load can be computed. In contrast to classical approaches this cost computation can handle asynchronism for message passing and communication/computation overlap.
\end{abstract}

Keywords: Cost Model; Symbolic Date; Parallel Programming Languages; Asynchronous Execution; Structural Clock.

\section{Introduction}

An important problem of parallel programming is to define a model of parallel complexity to a priori quantify the efficiency of an algorithm. Several complexity models have been proposed for synchronous computations, but the prevailing one is the PRAM model [3]. The cost associated to an algorithm is the number of synchronous elementary steps performed. The PRAM model allows straightforward cost evaluation for complex algorithms. Nevertheless, it is not well suited for actual distributed memory machines since it does not take into account communication overhead.

We defined in [5] a structured communication model called $\mathcal{S C L}$-chan. The communication semantics, driven by the syntactic structure of the program, yields a simple synchronous programming model and an efficient loosely synchronized execution model. In this paper, we show that it is possible to define a cost model for $\mathcal{S C} \mathcal{L}$-chan programs. We propose a complexity function that yields a symbolic date for each communication event. These dates can be ordered, allowing to compute, for each date, an upper bound for the network load. In contrast to classical approaches, such as BSP [4, 8], the cost computation can handle asynchronism for message passing and communication/computation overlap. \footnotetext{
Ressources en Informatique Scientifique", Orsay, France. Project 990829.

* This work has been partly supported by the "Institut du Développement et des 


\section{A Structured Message Passing Language}

The abstract machine is a distributed memory asynchronous parallel machine with $P$ processors. Processors are also called indices and denoted by $u, v, \ldots$ The value of a variable $X$ stored in the memory of an index $u$ is denoted by $\left.X\right|_{u}$. Data exchanges between indices take place through explicit send/receive. Data sent and not yet received are stored in channels handled as LIFO (Last-In First-Out) lists. There exists exactly one channel by variable and by pair of indices. We denote by $(u, v, X)$ the channel associated to the sender $u$, the receiver $v$ and the variable $X$. $\mathcal{S C L}$-chan programs rely on SPMD paradigm. Only the receive instruction can block, other instructions are executed asynchronously.

$X:=E$. An index $u$ updates its value $\left.X\right|_{u}$ in its memory with the value $\left.E\right|_{u}$. where $B$ do $S$ end. If an index $u$ evaluates the pure condition $\left.B\right|_{u}$ to true, then it executes the instruction block $S$.

$S ; T$. When an index finishes the execution of $S$, it starts the execution of $T$. forwhere $I:=A$ to $B$ do $S$ end. An index $u$ repeatedly executes $S$ until its counter $\left.I\right|_{u}$ reaches the value $\left.B\right|_{u}$.

send $X$ to $A$. This instruction is non blocking. A sending index $u$ sends the value $\left.X\right|_{u}$ to the index $v=\left.A\right|_{u}$, i.e. it adds this value into the channel $(u, v, X)$. receive $X$ from $A$ into $Y$. A receiving index $u$ waits that the sending index $v=$ $\left.A\right|_{u}$ has executed all the send instructions coming before (syntactically, unfolding the loops) this receive. Then, index $u$ extracts the last of the corresponding values from $(v, u, X)$.

\section{A Complexity Function for $\mathcal{S C} \mathcal{L}$-chan Programs}

We now define a complexity function for $\mathcal{S C L}$-chan programs. We first present the computation of the communication time of a datum. We model the network by an input and an output buffer for each index and a global router. First the sender copies the data from its memory into the output buffer. Then the router transmits the message from the output buffer of the sender to the input buffer of the receiver. Finally the receiver copies the message from its input buffer into its memory. The time required to execute an elementary instruction defines the time unit we use for all indices. To quantify the communications, we introduce a few architecture parameters. We denote by $\beta_{o}$ (resp. $\beta_{i}$ ) the start-up time and by $\tau_{o}$ (resp. $\tau_{i}$ ) the copy rate to access the output (resp. input) buffer. We denote by $\tau_{r}$ the transmission rate through the router. The transmission time of a message depends on the other messages currently in transit through the router. The communication time for a message $M$, assuming that $n$ messages $M_{1}, \ldots, M_{n}$ are currently in transit, is given by $\beta_{o}+\operatorname{size}(M) \tau_{o}+l \tau_{r}+\beta_{i}+\operatorname{size}(M) \tau_{i}$ with $l=\operatorname{size}(M)+\sum_{k=1}^{n} \operatorname{size}\left(M_{k}\right)$.

To model the execution of a $\mathcal{S C} \mathcal{L}$-chan program, we consider a weighted causality graph where each node (inst, $u$ ) corresponds to an instance of the instruction inst executed by the index $u$. The weight of each arrow corresponds 
to the minimum number of time units between the executions of the two corresponding instances. Note that these weights are symbolic expressions depending on the architecture parameters. We associate a symbolic execution date to each node by computing the longest path ending at this node. The execution cost for an algorithm $S$ denoted by $\operatorname{Cost}(S)$ is the maximum, for all indices, of the final execution dates.

To evaluate the load $l$, we first deduce from the causality graph a set $N e t$ gathering all sent messages labelled by their dates of emission. To each node corresponding to a sending instruction, we associate an element of $N e t$ defined as a pair composed by the date where the message enters the network and the size of the message. The entering date corresponds to the date of the node augmented by the time of copy to the output buffer. We denote by $l(d)$ the network load associated to the execution date $d$. The notation $l$ will be used instead of $l(d)$ in case the date $d$ is not ambiguous. The value of $l(d)$ corresponds to messages sent before $d$ and not yet received. To compute an upper bound for $l(d)$, we have to define an order on dates which subsumes all possibles execution orders. Let $d$ and $d^{\prime}$ be two symbolic dates. We say that $d$ is lower than $d^{\prime}$, denoted by $d<d^{\prime}$, if the expression $d^{\prime}$ is an upperbound for the expression $d$. Since two expressions may be not comparable, the order on dates is only a partial order. The load function $l(d)$ considers all messages with an entering date lower than $d$ or not comparable to $d$ and then, it selects all messages arrived at a date later than $d$ or not comparable to $d$.

$$
l(d)=\Sigma\left\{s /\left(s, d^{\prime}\right) \in N e t \wedge \operatorname{not}\left(d<d^{\prime}\right) \wedge \operatorname{not}\left(d^{\prime}+l\left(d^{\prime}\right) \tau_{r}<d\right)\right\}
$$

Example1. Let us consider the following program where each index sends a value to its neighbor.

$$
\begin{aligned}
& \text { where }(\text { This } \bmod 2)=0 \text { do; } X:=1 \text {; end; } \\
& \text { send } X \text { to }(\text { This }+1) \text {; receive } X \text { from }(\text { This }-1) \text { into } Y
\end{aligned}
$$

The left hand side of the following figure displays the corresponding causality graph. We suppose that the size of the message is 10 . We denote by $\delta(u)$ the final execution date for the index $u$. The max expression encodes the longest path search when a communication occurs.

$\delta(u)=\left\{\begin{array}{l}\beta_{o}+10 \tau_{o}+\max \left\{3,2+l\left(2+\beta_{o}+10 \tau_{o}\right) \tau_{r}\right\}+\beta_{i}+10 \tau_{i} \text { if } u \text { is even } \\ \beta_{o}+10 \tau_{o}+\max \left\{2,3+l\left(3+\beta_{o}+10 \tau_{o}\right) \tau_{r}\right\}+\beta_{i}+10 \tau_{i} \text { otherwise }\end{array}\right.$

The right hand side of the figure displays the ordering of the dates. We now construct the set $N$ et. As each odd (resp. even) index sends $X$ at the date $2+\beta_{o}+10 \tau_{o}\left(\operatorname{resp} .3+\beta_{o}+10 \tau_{o}\right)$. We obtain: $N e t=\left\{\left(2+\beta_{o}+10 \tau_{o}, 10\right), \ldots,(3+\right.$ $\left.\left.\beta_{o}+10 \tau_{o}, 10\right), \ldots\right\}$.

The load for the date $2+\beta_{o}+10 \tau_{o}$ have only to take into account the messages sent by the odd indices, we have $l\left(2+\beta_{o}+10 \tau_{o}\right)=5 P$. Since we cannot ensure that these messages exit the network before the date $3+\beta_{o}+10 \tau_{o}$, the load for the date $3+\beta_{o}+\tau_{o}$ has to take into account all the messages, then $l\left(3+\beta_{o}+10 \tau_{o}\right)=$ $10 P$. Thus $\operatorname{Cost}(S)=\max \{\delta(u)\}=3+\beta_{o}+10 \tau_{o}+10 P \tau_{r}+\beta_{i}+10 \tau_{i}$. 

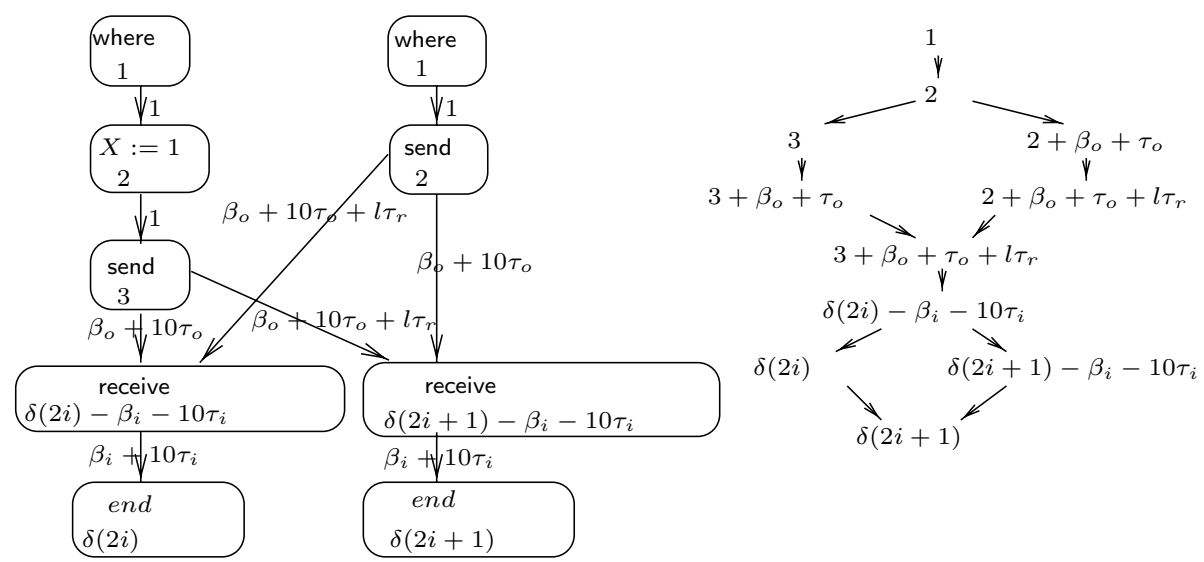

Example2. We now study a scan algorithm. The $N$ data are distributed by block. We demote by $M=N / P$ the size of each block. The algorithm can be divided into three parts as shown by the following array. As no communication occurs between two different parts, the corresponding instances of instructions can be labeled independently. We denote by $\delta_{\text {inst }}(u)$ the resulting date of the execution of the instance inst by the index $u$. The array displays the construction of the execution dates.

\begin{tabular}{|c|c|c|}
\hline Inst. & Program & $\delta_{\text {Inst }}(u)$ \\
\hline $2 I-3$ & forwhere $I:=2$ to $M$ do & $2 I-3$ \\
\hline $\begin{array}{l}2 I-2 \\
2(M-1)+1\end{array}$ & $\begin{array}{l}X[I]:=X[I]+X[I-1] \text {; } \\
\text { end; }\end{array}$ & $\begin{array}{l}2 I-2 \\
2(M-1)\end{array}$ \\
\hline 1 & $Y:=X[M]$ & $2 M-1$ \\
\hline & send $Y$ to This +1 & $2 M+\beta_{o}+\operatorname{size}(Y) \tau_{o}$ \\
\hline $4 I-1$ & forwhere $I:=1$ to $\left\lfloor\log _{2}(\right.$ This $\left.)\right\rfloor$ do & $\delta_{4 I-2}(u)+1$ \\
\hline $4 I$ & $\begin{array}{l}\text { receive } Y \\
\quad \text { from } T \text { his }-2^{(I-1)} \\
\text { into } Z\end{array}$ & $\begin{array}{l}\max \left\{\delta_{4 I-1}(u) ; \delta_{4 I-3}\left(u-2^{(I-1)}\right)+\right. \\
\left.\quad l\left(\delta_{4 I-3}\left(u-2^{(I-1)}\right)\right) \tau_{r}\right\}+ \\
\beta_{i}+\operatorname{size}(Y) \tau_{i}\end{array}$ \\
\hline $4 I+1$ & $Y:=Y+Z$ & $\delta_{4 I}(u)+1$ \\
\hline $\begin{array}{l}4 I+2 \\
4\left\lfloor\log _{2}(u)\right\rfloor+3\end{array}$ & $\begin{array}{l}\text { send } Y \text { to } \text { This }+2^{I} \\
\text { end; }\end{array}$ & $\begin{array}{l}\delta_{4 I+1}(u)+\beta_{o}+\operatorname{size}(Y) \tau_{o} \\
\delta_{4\left\lfloor\log _{2}(u)\right\rfloor+2}(u)\end{array}$ \\
\hline \begin{tabular}{l|}
$2 I-1$ \\
$2 I$ \\
$2 M+1$ \\
\end{tabular} & $\begin{array}{l}\text { forwhere } I:=1 \text { to } M \text { do } \\
X[I]:=X[I]+Y-X[M] \\
\text { end }\end{array}$ & $\begin{array}{l}\delta_{4\left\lfloor\log _{2}(u)\right\rfloor+2}(u)+2 I-2 \\
\delta_{4\left\lfloor\log _{2}(u)\right\rfloor+2}(u)+2 I-1 \\
\delta_{4\left\lfloor\log _{2}(u)\right\rfloor+2}(u)+2 M \\
\end{array}$ \\
\hline
\end{tabular}

In the second part of the algorithm, communications occur. We first construct the set $N e t: N e t=\left\{\left(\delta_{4 I+2}(u), \operatorname{size}(Y)\right) / u \in[1 ; P] \wedge I \in\left[0 ; \log _{2}(u)\right]\right\}$. Since we have $\delta_{4 I-2}\left(u-2^{(I-1)}\right)<\delta_{4 I+3}(u)$, the network load of a given iteration only has to take into account the messages sent during the previous iteration. We obtain $l(2 M+1)=P$ size $(Y)$ and $l\left(\delta_{4 I+2}(u)\right)=\left(P / 2^{I}\right)$ size $(Y)$. By induction on the iterations of the forwhere loop we obtain the final date $\delta(u)=4 M+\beta_{o}+$ $\tau_{o}+\log _{2}(u)\left[2+\beta_{i}+\beta_{o}+\operatorname{size}(Y)\left(\tau_{i}+\tau_{o}\right)\right]+\left(2-2^{-\left\lfloor\log _{2}(u)\right\rfloor-1}\right) P \operatorname{size}(Y) \tau_{r}$, and hence $\operatorname{Cost}(S)=\max \{\delta(u)\}=\delta(P)$.

By using an implementation of $\mathcal{S C} \mathcal{L}$-chan on a Cray T3E, we measured the values of parameters via a ping pong test repeated one hundred times. We have estimated the cost of the scan on a Cray T3E for 32 processors. Then we measured the execution time. The following array displays results in $\mu s$. 


\begin{tabular}{|l|l|l|l|l|}
\hline$N$ (word) & 64 & 320 & 3200 & 32000 \\
\hline Cost $(S)\left(10^{-6}\right.$ sec. $)$ & 756 & 756 & 757 & 759 \\
\hline Measured $(S)\left(10^{-6}\right.$ sec. $)$ & 694 & 701 & 718 & 792 \\
\hline
\end{tabular}

\section{Conclusion}

We presented a complexity model well suited for asynchronous structured message passing. Thanks to our structured communication semantics, we were able to build a complexity time function relying on the program structure and on the sequential complexity on each index. It yields a symbolic expression encoding the communication scheme. This result is used to evaluate an upper bound for the network load taking into account the asynchronism of communication events. By using this evaluation, we can deduce a symbolic cost associated to a given execution of the algorithm. Note that our model is general enough to be easily adapted to another modelization of the network, for example by introducing several routers or an adaptative routing.

In contrast to models like BSP, CGM, MINIBSP [2, 4], 7], 8], asynchronous communication-computation overlapping can be taken into account. In contrast to LogP 1],structured communication schemes can allow to compute worst case costs [6].

Experimental results on Cray T3E highlight accurate cost predictions. The complexity model appears to be a good trade-off between usability and effectiveness.

\section{References}

[1] D. Culler, R. Karp, D. Patterson, A. Sahay, K. E. Schauser, E. Santos, R. Subramonian, and V. E. Thorsten. LogP: Towards a realistic model of parallel computation. In Proceedings of the Fourth ACM SIGPLAN Symposium on Principles 8 Practice of Parallel Programming, pages 1-12, May 1993.

[2] F.Dehne, A.Fabri, and A. Rau-Chaplin. Scalable Parallel Geometric Algorithms for Coarse Grained Multicomputer. ACM 9th Symposium on Computational Geometry, 1993.

[3] J. Gabarro J. Balcazar, J. Diaz. Structural Complexity II. Springer-Verlag, 1989.

[4] W F McColl. General purpose parallel computing. In A M Gibbons and P Spirakis, editors, Lectures on Parallel Computation. Proc. 1991 ALCOM Spring School on Parallel Computation, pages 337-391. Cambridge University Press, 93.

[5] Emmanuel Melin, Bruno Raffin, Xavier Rebeuf, and Bernard Virot. A Structured Synchronization and Communication Model Fitting Irregular Data Accesses. Journal of Parallel and Distributed Computing, 50(1):3-27, 1998.

[6] Emmanuel Melin, Bruno Raffin, Xavier Rebeuf, and Bernard Virot. A Cost Model For Asynchronous and Structured Message Passing (Extended Version). Technical Report RR99-07, LIFO, Orléans, France, April 1999.

[7] D. B. Skillicorn. miniBSP: A BSP Language and Transformation System. http://www.qucis.queensu.ca/home/skill, 1996.

[8] L. G. Valiant. A Bridging Model for Parallel Computation. Communications of the ACM, 33(8):103-111, August 1990. 